\title{
Neuroimmune disorders of the central nervous system in children in the molecular era
}

Elizabeth Wells ${ }^{*}$, Yael Hacohen ${ }^{2 *}$, Amy Waldman ${ }^{3}$, Jan M Tillema ${ }^{4}$, Ariane Soldatos ${ }^{5}$, Beau Ances $^{6}$, Susanne Benseler ${ }^{7}$, Bibi Bielekova ${ }^{8}$, Russel C Dale ${ }^{9}$, Josep Dalmau ${ }^{10}$, William Gaillard, $\mathrm{MD}^{1}$, Mark Gorman ${ }^{11}$, Benjamin Greenberg ${ }^{12}$, AnnHyslop ${ }^{13}$, Carolos A Pardo ${ }^{14}$, Robert C.RC Tasker $^{15}$, E. Ann Yeh ${ }^{16}$, Amit Bar-Or ${ }^{17}$, Sean Pittock ${ }^{4,18}$, Adeline Vanderver ${ }^{3 \#}$, Brenda Banwell ${ }^{3 \#}$

* these authors are joint first authors

\# these authors are joint senior authors

1. Children's National Health System, Center for Neuroscience and Behavioral Medicine, Washington, DC, USA

2. Department of Neuroinflammation, Queen Square MS Centre, UCL Institute of Neurology. Paediatric Neurology, Great Ormond Street Hospital, London, UK

3. The Children's Hospital of Philadelphia, Department of Neurology and Pediatric Multiple Sclerosis Clinic, Philadelphia, PA, USA

4. Mayo Clinic, Department of Neurology, Rochester, MN, USA

5. National Institutes of Health, National Institute of Neurological Disorders and Stroke, Bethesda, MD, USA

6. Washington University in St. Louis, Department of Neurology, St. Louis, MO, USA

7. Section of Rheumatology, Department of Pediatrics, Alberta Children's Hospital,Calgary Alberta, Canada

8. Neuroimmunological Diseases Unit, National Institute of Neurological Disorders and Stroke, National Institute of Health, Bethesda, MD, USA

9. University of Sydney, Institute for Neuroscience and Muscle Research, The Kids Research Institute at the Children's Hospital at Westmead, Westmead NSW, Australia

10. ICREA-IDIBAPS, Hospital Clínic, University of Barcelona, Spain; and Department of Neurology, University of Pennsylvania. , USA

11. Boston Children's Hospital, Department of Neurology, Boston, MA, USA

12. University of Texas Southwestern, Department Neurology \& Neurotherapeutics, Pediatrics, Dallas, TX, USA

13. Miami Children's Hospital, Department of Pediatric Neurology and Epilepsy, Miami, FL, USA

14. Johns Hopkins Director of the Johns Hopkins Transverse Myelitis Center, Baltimore, MD, USA

15. Boston Children's Hospital, Department of NeuroCritical Care, Boston MA, USA

16. University of Toronto, The Hospital for Sick Children, Department of Pediatrics (Neurology), Toronto, ON, USA

17. McGill University, Montreal Neurological Institute and Hospital, Department of Neurology and Neurosurgery, Montreal, QC, USA

18. Mayo Clinic, Department of Laboratory Medicine and Pathology, Rochester, MN, USA 


\section{Abstract}

Immune-mediated disorders of the CNS in children are a complex group of demyelinating, inflammatory, parainfectious and postinfectious disorders with heterogeneous pathobiological mechanisms and clinical manifestations, often associated with fundamental derangement in immune regulation. In this Review, we aim to provide an update on our knowledge of neuroimmune disorders and highlight areas of research that are priorities for improving clinical management. We outline the clinical features of neuroimmune disorders, the current approaches to their treatment and new approaches in development. We then consider the pathological features, including biomarkers, pathological mechanisms and genetics, and discuss the value of immune assays in clinical investigation and basic research. On the basis of current knowledge and techniques, we propose four research priorities: rigorous and consistent collection of core clinical data, co-operative investigation of treatments, development of biological assays, and genetic studies. These priorities should help us to achieve the shared goal of precision medicine for neuroimmune disorders. However, multicentre research and the creation of clinical consortia for these rare disorders will be necessary, and we hope that this Review serves as a call to action that is timely given current exciting advances in neuroimmune therapeutics.

\section{Introduction}

Neuroimmune disorders are a heterogeneous group of severe neurological conditions primarily characterized by inflammatory responses in the CNS ${ }^{1}$. Many of these disorders manifest most commonly in childhood or adolescence, whereas others more typically occur in adults ${ }^{2,3}$. Although all of these disorders share heightened immune responses against the CNS, the underlying pathobiology differs; cell-mediated, humorally mediated, infection-triggered and genetically defined mechanisms are each involved in different disorders.

Despite increased clinical recognition of neuroimmune disorders and increasingly sophisticated diagnostic techniques, specific neuroimmune disorders remain rare, or are rare in children (as in the case of MS), although they are not uncommon when considered as a group of disorders. Consequently, multicentre collaborative research is essential for the identification and study of well-characterized patients that is necessary to develop precision medicine and targeted immune therapies. Collaborative research studies in which paediatric-onset and adultonset manifestations of the same neuroimmune disease are compared could reveal patterns of clinical presentation and biological mechanisms that drive age of onset. Such comparisons 
might also delineate whether clinical differences are mediated by age-related immune responses, age-related exposures or a differential impact on the maturing CNS.

In this Review, we provide an update on the current knowledge about the clinical features of neuroimmune disorders and approaches to their management. We then review the pathobiology of neuroimmune disorders, including pathological markers, pathological mechanisms and genetics, and propose areas of research that are priorities if targeted treatment approaches are to be successfully developed. We focus on disorders that affect the CNS specifically, and consequently do not discuss neurological disorders in the context of systemic autoimmune diseases. We hope that the Review will serve as a call to action to harness the increasing array of new therapeutic agents that hold promise for children with these severe disorders.

\section{[H1] Features of neuroimmune disorders}

\section{[H2] Clinical features}

Most neuroimmune disorders occur in previously healthy, developmentally and neurologically normal children and adolescents. The onset of neurological deficits is abrupt in most patients, although a prodrome (for example, fever, malaise, mild infectious symptoms or psychiatric symptoms) can develop several weeks before neurological symptoms. Characterization of neuroimmune disorders is aided by dividing neurological features at presentation into those that localize to a single CNS site (clinically monofocal) and those that involve multiple CNS regions (polyfocal); patients with polyfocal deficits can be further divided into those with encepalopathy and those with preserved alertness. Traditionally, neuroimmune disorders have been stratified into disorders that target the white matter, known as acquired demyelinating syndromes (ADS), and disorders that predominantly target the grey matter, which are classed as antibodymediated autoimmune encephalopathies. More recently with advanced in diagnostics there is a shift to stratify of syndromes (beyond the clinical and radiological phenotypype) according to the underlying pathogenic mechanism. Specific infections are rarely identified. Figures $1 \mathrm{a}$ and $1 \mathrm{~b}$ summarize the key features of many of the currently recognized neuroimmune conditions and Figure 2 illustrates MRI findings. In some patients, particularly those with genetic defects in specific immune pathways, disease onset can occur in infancy and can be associated with very early onset CNS impairment.

Neuroimmune conditions can be grouped according to the putative primary immune mechanism (Supplementary information S1 (table)) but more than one immune mechanism 
might be involved, and the pathogenicity of antibodies that have been associated with some disorders has not yet been proven. Inflammation in the CNS is clearly present in some disorders, but whether immune activity is causal or secondary remains to be fully elucidated for many conditions. A unifying aspect of these disorders is that the disease course can be modulated by immune-targeted therapies.

As many neuroimmune disorders can be phenotypically similar to other inflammatory and noninflammatory conditions, deep phenotyping that includes the temporal profile of symptoms, specific radiological and immunological parameters and exclusions of alternative diagnoses is required in clinical practice to be sure of the diagnosis. An intriguing aspect of these antibody-mediated disorders is the variation in the presence of detectable antibodies according to demographics ${ }^{4}$. For example, most paediatric patients who are positive for aquaporin 4 (AQP4) antibodies are post-pubescent females, and these antibodies are not common among younger children. By contrast, antibodies against myelin oligodendrocyte glycoprotein (MOG) are more frequently detectable in children than in adults. Anti-NMDA receptor antibodies are most commonly detectable in females aged 10-40 years, and are at least as common among children as among adults. Other antibodies against neuronal targets, such as those detected in adults with limbic encephalitis ( $L G 1$, AMPA receptor and GABA receptor antibodies) have been reported only in isolated cases and the clinical manifestations can be variable ${ }^{3}$. When results of antibody assays are discordant with the phenotype or when the diagnosis is made in an atypical demographic group, the clinical phenotype should remain the gold standard for diagnosis. Furthermore, different pathogenic mechanisms can contribute to different stages of disease; in this case, the acute triggers need to be distinguished from the mechanism driving chronic inflammation. Alternatively, patients can develop more than one disease: for example, patients with anti-NMDA receptor encephalitis, which is not typically associated with demyelination, can develop a second distinct episode with clinical and MRI features of CNS demyelination secondary to AQP4 or MOG antibodies ${ }^{5,6}$.

\section{[H2] Neuroimaging features}

$\mathrm{MRI}$ is important in the investigation of suspected neuroimmune disorders in children (Fig. 2). Neuroimmune disorders typically share MRI features such as focal areas of hyperintensity on T2-weighted MRI ("lesions"), regional loss of tissue on T1-weighted imaging ("black holes") and loss of brain, optic nerve and spinal cord volumes ${ }^{7}$. However, normal MRI findings do not exclude a neuroimmune disorder; for example, in many patients with anti-NMDA receptor encephalitis, the brain looks normal on $\mathrm{MRI}^{8}$. 
When MRI findings are abnormal, pattern analysis is valuable for diagnosis of many neuroimmune disorders ${ }^{9,10}$. For example, the pattern of lesions is key to distinguishing between MS and neuromyelitis optica spectrum disorder (NMOSD). In MS, focal lesions are predominantly juxtacortical and periventricular, and those that are in the posterior fossa or spinal cord tend to be small ${ }^{11}$. By contrast, in NMOSD, lesions in the brain are often in the diencephalon and periaqueductal grey matter, and lesions that involve the spinal cord are longitudinally extensive (spanning more than three spinal segments) ${ }^{9,10}$. More-advanced MRI techniques and higher strength magnets can reveal a greater extent of tissue abnormalities, including changes in the brain or leptomeninges, that are not detected by conventional T2weighted sequences ${ }^{12}$. For example, myelin water imaging, a magnetic resonance measure of myelin content that evaluates the amount of intramyelinic and extracellular water, can differentiate between AQP4-antibody-associated NMOSD, which is an astrocyopathy, and MS, in which macrophage-mediated phagocytosis leads to loss of myelin ${ }^{13}$. Whether diffusion tensor imaging and magnetization transfer imaging, which provide information about axonal integrity and the extent of myelination, can assist with distinguishing between different neuroimmune disorders is not currently known.

PET imaging can also be valuable for assessment of patients with neuroimmune disorders. ${ }^{18} \mathrm{~F}$-deoxyglucose (FDG)-PET imaging can reveal focal hypermetabolism, particularly in mesial temporal structures, in patients with autoimmune encephalitis; evidence suggests that, at least in some patients, this hypermetabolism results from subclinical seizure activity ${ }^{14}$. Use of the PET ligand ${ }^{11} \mathrm{C}-\mathrm{PBR} 28$, which recognizes an $18 \mathrm{kDa}$ translocator protein that is expressed in activated microglia and macrophages, has indicated increased activation of these immune cells in multiple brain regions in adults with $M S,{ }^{15}$ although the value of this technique in MS remains controversial.

As is true for the clinical course, MRI changes over time aid in confirming the diagnosis of different neuroimmune disorders. Accrual of new focal lesions is a hallmark of $\mathrm{MS}^{11}$. In patients with AQP4-antibody-associated NMOSD and MOG-antibody-associated demyelination, new lesions are typically seen only at the time of clinical relapse, and considerable resolution of the lesions can be seen in follow-up scans ${ }^{16}$. The interferonopathy Aicardi-Goutières syndrome (AGS) is characterized by increasing calcium deposition over time and progressive brain atrophy ${ }^{17}$, and progressive hemi-hemispheric atrophy is a key criterion for the diagnosis of Rasmussen encephalitis ${ }^{18}$.

\section{[H2] Clinical biomarkers}


Analysis of CSF and/or serum from people with a suspected neuroimmune disorder is currently used for the detection of specific antibodies (Supplementary Table 1), exclusion of infection and evaluation of markers of neuroinflammation. Circulating immune biomarkers might not reflect the intrathecal environment, so evaluation of cerebrospinal fluid (CSF) as well as serum is typically essential. For example, serum and CSF levels of antibodies differ markedly between individual patients with NMOSD or anti-NMDA receptor encephalitis ${ }^{19-21}$. Sequential testing of serum and then CSF if the serum is negative should be discouraged, as this practice can delay diagnosis and therapy ${ }^{3}$.

Several possible biomarkers have potential for clinical assessment and monitoring of neuroimmune disorders. One example is CSF levels of neopterin, a marker of $T_{H} 1$ activation that is produced by lymphocytes, macrophages, dendritic cells and neurons after stimulation by interferon and has been suggested as a nonspecific marker of CNS inflammation ${ }^{22}$. Measurement of interferon responses in peripheral blood by use of interferon-stimulated gene panels might also prove valuable in monitoring CNS inflammatory responses and responses to anti-inflammatory therapy in patients with neuroimmune disorders ${ }^{23}$. In patients with suspected interferonopathies, evaluation of the expression of interferon-stimulated gene panels in the blood and evaluation of CSF neoptrin levels can be useful diagnostically while waiting for genetic confirmation or when tests for the known AGS genetic mutations are negative ${ }^{23}$. CSF levels of markers of neuronal damage (for example, tau, neuronal specific enolase, $S 100 \beta$ and neurofilament light chain) indicate neuronal injury, although none of these markers are diseasespecific ${ }^{24}$.

\section{[H1] Treatment of neuroimmune disorders}

\section{[H2] Symptomatic management}

Some children with neuroimmune disorders require intensive care, including a temporary endotracheal tube or nasogastric tube, and are at risk of serious complications, including thromboembolism, infection and skin breakdown ${ }^{25}$. Comprehensive multidisciplinary care is essential. Symptomatic treatment of seizures, movement disorders and dysautonomia can hasten discharge from the intensive care unit, enable earlier participation in rehabilitation, and prevent secondary conditions, such as friction ulcers, contractures and iatrogenic infections ${ }^{26,27}$.

As awareness of immune mechanisms in paediatric epilepsies increases, greater numbers of patients are now treated with immunotherapy in addition to anti-epileptic drugs ${ }^{28}$. Prolonged electroencephalographic monitoring can help to identify subtle or electrographic 
seizures (in addition to specific patterns such as the extreme delta brush seen in patients with anti-NMDA receptor encephalitis) that need anti-epileptic therapy until the immune disease is controlled $^{29}$.

Following acute care, many children require inpatient rehabilitation. Excellent outcomes have been reported after prolonged rehabilitation therapy after anti-NMDA receptor encephalitis, with clinical improvement still emerging $>2$ years after disease onset ${ }^{25}$.

\section{[H2] Disease-targeted treatment}

Specific disease-targeted treatment strategies have been proposed for adults and children with neuroimmune disorders ${ }^{3,30}$. Therapies include acute strategies and strategies to prevent relapses. Initial treatment for most neuroimmunological diseases includes corticosteroids (intravenous methylprednisolone and/or oral prednisolone), intravenous immunoglobulin (IVlg) or plasma exchange ${ }^{31}$. Plasma exchange is usually reserved for when intravenous methylprednisolone with or without IVIg has been only partially effective, but it can be considered at an earlier stage, especially for patients with a known, proven autoantibodymediated disorder, such as AQP4 antibody associated NMOSD. A response to acute immunotherapy can serve as a 'proof of principle' diagnostic test when a neuroimmune disorder is suspected but not confirmed (for example, when results of antibody testing are pending) ${ }^{32}$. The mechanisms of action of IVIg and steroids include immunosuppressive and immunomodulative effects. These therapies could also benefit patients with primary genetic conditions and secondary inflammation, such as that seen in patients with the mitochondrial disorder DARS-associated leukoencephalopathy, who respond to steroids ${ }^{33}$.

Acute administration of high-dose corticosteroids can be delayed by concerns that the patient has a CNS infection. CSF analysis can be used to exclude specific infections, such as herpes simplex virus (HSV). Emerging next-generation sequencing approaches, such as metagenomics analysis to detect non-human nucleic acid in the CSF or brain tissue, can provide faster results and, when negative, reduce the concerns of corticosteroid use. However, the risk of infectious propagation as a result of corticosteroid use when CNS infection has not been excluded is unclear, and corticosteroids are of proven benefit when combined with antiinfectious therapies for the specific brain infections (as in meningococcal infection ${ }^{34}$ and CNS tuberculosis ${ }^{35}$. A study of viral replication in cell cultures that were transfected with HSV demonstrated that dexamethasone was associated with increased viral reactivation rate, but this effect was abolished if dexamethasone was applied in conjunction with the antiviral acyclovir ${ }^{36}$. Furthermore, a systematic review of studies that included 43 patients with HSV-induced anti- 
NMDA receptor encephalitis found no reports of HSV reactivation despite the fact that $93 \%$ of patients received immunosuppressive therapies ${ }^{37}$.

The choice of immunosuppressive therapy is influenced by the underlying diagnosis, disease pathobioology, disease severity and the need for a rapid onset of immunosuppression. International consensus guidelines for the treatment of paediatric MS have been published ${ }^{38}$, and cohort studies have included descriptions of treatment models for paediatric AQP4antibody-associated NMOSD ${ }^{39}$ and MOG-antibody-associated disease ${ }^{40}$. Rituximab and cyclophosphamide have a rapid onset of action, and have been used in patients with severe neuroimmune conditions in the hope of inducing rapid clinical remission ${ }^{39}$. For most neuroimmunological diseases, treatment regimens have not been established and neither have evidence-based means to categorize patients according to the likelihood that they will require chronic treatment.

After standard acute therapy, the decision of whether ongoing therapy is needed is typically influenced by the response to the initial therapy, the likelihood of continued CNS inflammation (for example, anti-NMDA receptor encephalitis is a monophasic disease in the majority) and the risk of relapse (for example, in relapsing conditions such as MS or NMOSD).

\section{[H2] Clinical trials}

Most neuroimmune disorders are too rare for therapies to be tested in clinical trials, but trials of therapeutic agents for paediatric MS (one clinical trial completed, several ongoing), AGS (phase II trial enrolling) and NMOSD (consensus guidelines for treatment being proposed) are being actively pursued. Results from randomized controlled trials of phenytoin ${ }^{41}$ and erythropoietin ${ }^{42}$ in optic neuritis suggest that neuroprotective agents as well as immunotherapy are beneficial in acute demyelination.

All of these trials are restricted by the rarity of the conditions, and investigators and legislators must also be sensitive to the risk that placebo-controlled trial designs might be unacceptable because even a single relapse could cause patients severe deficits and because subclinical insults could be ongoing. Treatment algorithm models, akin to many protocols used in paediatric oncology trials, are worth considering for trials in neuroimmune disorders. Such models are consensus-based and adhered to by all partnering institutions, they incorporate structured inclusion, exclusion and outcome metrics, and the biological materials for evaluation are collected with a consistent protocol. Nevertheless, post-hoc retrospective analysis of clinically acquired data can provide important observations about the treatment responses and enable inclusion of a larger number of patients. For example, a multicentre study that included 
577 children and adults with anti-NMDA receptor encephalitis ${ }^{25}$ revealed that first-line immunotherapy is often insufficient and the addition of second-line immunotherapy is associated with better outcomes. Simillarly, a multicentre, multinational study of rituximab use in the treatment of paediatric neuroimmune disorders demonstrated that earlier use of rituximab was associated with greater functional improvement ${ }^{39}$.

\section{[H2] Risks of treatment}

Treatment of neuroimmune conditions, particularly with concurrent or sequential immunotherapy, requires knowledge and careful consideration of the risk of toxicity and adverse events. The potential for long-term sequelae, including the effects of childhood immunosuppression on subsequent fertility, the risk of malignancy and the possibility of premature immune senescence, necessitates long-term outcome studies and care that focuses on 'late effects'. Large retrospective studies in which the safety of specific immunotherapies in paediatric neuroimmune disorders was assessed have provided some estimates to aid clinical decision making ${ }^{39,43}$ (for example, a 7.6\% rate of infection with rituximab), and future work could include the establishment of large databases and systematic prospective reporting.

Chronic administration of steroids has adverse effects on muscle, bone, mood and the endocrine system ${ }^{44}$, and steroid-sparing medications, which achieve systemic immunosuppression without the steroid-related side effects, such as azathioprine or mycophenolate mofetil might need to be considered if patients have recurance of disease activity when tapering of steroids is attempted.

\section{[H2] The future of treatment}

Innovative imaging, neuropathological and cell-based diagnostic approaches have led to an explosion of research into the molecular underpinnings of neuroimmunological disease and the rational design of molecular therapies to deliver precision medicine. Monoclonal antibodies are rapidly emerging, and include different models of treatment for $\mathrm{MS}^{45}$, refinement of $\mathrm{B}$ cell therapies in $\mathrm{NMOSD}^{46}$, blocking of specific cytokine production (for example, IL-1 $\beta$ for interferonopathies ${ }^{47}$ and anti-IL-6 receptor antibody tocilizumab in NMOSD ${ }^{48}$. These advances in molecular therapies means that the current clinical picture in neuroimmune disorders could be improved by a greater understanding of the pathobiological mechanisms, genetics and biomarkers. In the remainder of this Review, we focus on these pathobiological aspects and how improved understanding could lead to better clinical management. 


\section{[H1] Markers of pathology}

Levels of circulating immune cells, serum antibodies, cells in the CSF, intrathecal antibodies and brain pathology are all key markers of various neuroimmune conditions, and are important in distinguishing these conditions from other CNS diseases. As an example of the importance of such markers, the presence of MOG antibodies in the serum largely identifies children with demyelinating conditions distinct from $\mathrm{MS}^{49,50}$. In a study of 110 children with a relapsing acquired demyelinating syndrome (ADS), MOG and AQP4 antibodies were detected in $83 \%$ of children with phenotypes that were not typical of $\mathrm{MS}^{51}$. Children who tested positive for MOG antibodies exhibited notable and distinctive clinical and MRI features that differed from those seen in AQP4 antibody associated NMOSD and MS ${ }^{51}$.

Levels of these biomarkers can also vary throughout the disease course or their presence might be only transient in monophasic neuroimmune conditions, so serial evaluation is informative in a clinical context and can inform prognosis. In a study of patients with anti-NMDA receptor encephalitis, antibody titres in the CSF and serum at onset were higher in patients who went on to have poor outcomes, and were higher in patients with a teratoma than in patients with no tumours, suggesting that antibody titres (particularly in the CSF) can complement the clinical assessment ${ }^{52}$. However, antibody titres can remain high even after clinical recovery ${ }^{53}$. Similarly, persistence of a very high antibody titre is associated with a relapsing disease course in patients with MOG-antibody-associated disease ${ }^{54}$ or AQP4-antibody-associated disease ${ }^{55}$. In general, although antibody titres are higher during relapse than during remission, the absolute antibody titre cannot be used as a precise metric of disease status at the individual patient level$\left.\right|^{56}$.

Neuroimmune disorders can be caused by dysregulation of immune cells rather than by the production of pathogenic antibodies, so for some conditions, evaluation of circulating immune cell profiles can be informative. The relative proportion of immune cell subtypes (regulatory and effector $\mathrm{T}$ cells ${ }^{57}$ ), reduced telomere length in regulatory $\mathrm{T}$ cell populations $\mathrm{s}^{58}$, and patterns of CNS epitopes in the CSF identified by antigen array assays ${ }^{59}$ have all been measured in research studies of paediatric MS. Collectively, these studies indicate abnormalities in effector and regulatory $T$ cell subsets, and possibly immune senescence, in children with MS ${ }^{60}$. Few studies have addressed the immune cell profile of other neuroimmune disorders, at least in part owing to the complexity of the necessary assays. 
Although immunological signatures are diagnostically and prognostically valuable, the absence of detectable antibodies or specific immune cell phenotypes does not exclude a neuroimmune disorder (as defined by clinical criteria and supported by clinical responsiveness to immunosuppressive therapy). In a study of 164 Australian children with acute encephalopathy, an immune-mediated encephalitis was identified in only $34 \%{ }^{61}$. Positivity for antibodies was more common among individuals with concomitant movement disorders, psychiatric features and sleep disorders. Importantly, the clinical features of autoimmune encephalitis can occur in patients who are seronegative for autoantibodies when tested with currently available antibody panels, and these patients might still benefit from treatment. In a study of 48 children with autoimmune encephalitis, treatment with immunotherapies was associated with improved acute and long-term outcomes in patients who were seronegative for establish antibodies $^{62}$. Antibodies against neuronal and glial targets were also found in 22 of 57 children with brainstem encephalitis; in addition to neurological deficits consistent with brainstem involvement, the clinical features of the conditions in these children were often those associated with the specific antibodies identified ${ }^{63}$.

Diagnosis of neuroimmune disorders can also be made on the basis of CNS tissue examination. For example, a brain biopsy is the only means of confirming a diagnosis of isolated small vessel vasculitis ${ }^{64,65}$. Diagnosis requires evidence of perivascular inflammation, disruption and hyalinization of the vascular endothelium, and evidence of transmural extravasation of inflammatory cells ${ }^{65}$. The diagnostic value of a brain biopsy in general is strongly influenced by whether the tissue is taken from an area that appears abnormal on MRI and whether a full thickness biopsy that contains meninges, cortex and underlying white matter is obtained. Corticosteroid therapy before the biopsy can dramatically reduce the diagnostic yield ${ }^{66}$. Brain biopsies are typically only performed in children with acquired demyelination if they have tumefactive demyelination or when the clinical and radiological features are not atypical and the biopsy results could guide treatment.

\section{[H1] Pathobiological mechanisms [H2] Triggers of Immune Activation}

Onset of most neuroimmune disorders is acute or subacute, indicating environmental triggers of the aberrant immune response. Several neuroimmune disorders occur in close temporal proximity to infection; some disorders are directly related to interactions between host genetic factors and specific pathogens and others (for example, post-streptococcal Sydenham chorea) are presumed to result from molecular mimicry of CNS antigens by the pathogen. 
Exclusion of active CNS infection is essential because the acute features of neuroimmune disorders can be difficult to distinguish from infective encephalitis ${ }^{62,67,68}$. However, recent CNS infection can have an important role, as is the case in children who develop anti-NMDA receptor encephalitis after recovery from herpes simplex virus encephalitis (HSVE) ${ }^{69}$. This phenomenon was initially thought to be rare, but careful observation of children recovering from HSVE has indicated that up to $25 \%$ of patients develop a distinct subsequent illness characterized by encephalopathy and movement disorder ${ }^{70-72}$.

The mechanisms by which HSVE leads to anti-NMDA receptor encephalitis require elucidation. A conceivable mechanism is exposure of the immune system to new CNS antigens as a result of CNS damage from HSVE, leading to immune-directed attack of the CNS. However, if this hypothesis is true, anti-NDMA receptor encephalitis might be expected to develop after CNS trauma, stroke or other CNS insults but does not. Next-generation sequencing of immune cells in such patients might be informative, as it could identify upregulation of genes within specific relevant pathways.

Other examples of infection-related neuroimmune disorders include the development of CNS vasculitis after varicella zoster virus $(\mathrm{VZV})$ infection ${ }^{73}$, although the incidence of this condition is now decreasing with the launch of widespread vaccination against VZV74. The viral repertoire experienced during early life influences the risk of MS: remote infection with EpsteinBarr virus $^{75}$ is associated with an increased risk ${ }^{76}$. Environmental contributions, such as low vitamin $\mathrm{D}$ levels, exposure to cigarette smoke, obesity and dietary effects on the gut microbiome have also all been implicated as being permissive to MS pathobiology ${ }^{77}$.

Some immune-mediated encephalopathy syndromes are triggered by oncological processes and are part of paraneoplastic neurological disorders (Supplementary Table 1). Therefore, extensive investigations to rule out malignancy are advisable for certain patients, particularly older adolescent girls and young women with anti-NMDA receptor encephalitis, 40$60 \%$ of whom are likely to have an ovarian teratoma ${ }^{78}$, and children with opsoclonus myoclonus syndrome (characterized by opsoclonus, myoclonus, and ataxia, usually accompanied by behavioral abnormalities) of whom $\sim 50 \%$ will have neuroblastoma and/or other neural crest tumours. Refractory cases of autoimmune encephalitis in which no tumour has been detected warrant extensive evaluation for occult malignancy; in one case study of a patient with antiNMDA receptor encephalopathy, microscopic teratomas that were not seen on imaging were identified, and improvements were seen after oophorectomy ${ }^{79}$.

\section{[H2] Extracellular and intracellular antigens}


Beyond triggering events, the mechanisms by which immune responses mediate neurological disease are also important to understand. Classification of antibody-mediated neuroimmune disorders according to whether the antigen binding occurs on the extracelullar domain or intracellular domain of neuronal or glial receptors has both diagnostic and therapeutic importance. Antibodies that bind to the extracellular domains of CNS ion channels, receptors and other synaptic proteins are now recognized as a likely cause of neurological disorders in adults and children. These cell-surface antigens are often essential to cellular function or neurotransmission and are generally expressed throughout the nervous system. The pathogenic mechanisms are different for different autoantibodies, but patients with these disorders respond to removal of the antibodies with immunotherapies. By contrast, antibodies against intracellular antigens, as seen in most paraneoplastic syndromes and in the case of glutamin acid decarboxylase 65 (GAD-65), are generally considered to be unlikely initiators of disease and more likely to result from immune recognition of tissue destruction. These antibodies, although not directly pathogenic, could still contribute to propagation of established disease (relapse, progression) by T-cell-mediated mechanisms. More generally, in antibody-mediated disorders, upregulation in the $T_{H} 17$ pathway and raised CSF IL- 6 has been commonly observed, [a finding that is rare in $\mathrm{MS}^{80}$.

\section{[H3] Extracellular antigens}

Antibodies against cell-surface antigens that are associated with neuroimmunological disorders in children can be stratified into those that are associated with demyelinating phenotypes (AQP4 and MOG antibodies) and those that are associated with autoimmune encephalitis (anti-NMDA receptor and anti-GABA $A_{A}$ receptor antibodies). Autoantibodies against extracellular synaptic proteins that are associated with autoimmune encephalitis in adults ${ }^{81}$ are rare in children and have only been described in isolated case reports ${ }^{1,82}$.

Pathological studies in mice and humans have indicated that anti-APQ4 antibodies are directly pathogenic in NMOSD ${ }^{83}$. These antibodies are predominantly of the immunoglobulin $\mathrm{G} 1$ (IgG1) subtype and bind to 3D conformational epitopes on the extracellular loops of AQP4. This binding leads to astrocyte damage through complement-dependent cytotoxicity and subsequent loss of AQP4 expression. Given that astrocyte processes are also important to the integrity of the blood-brain barrier, AQP4-antibody mediated astrocyte damage also leads to blood-brain barrier disruption, causing leukocyte infiltration and cytokine release that results in damage to oligodendrocytes, myelin and neurons ${ }^{84}$. In anti-AQP4 antibody associated disease, CSF cytokine levels correlate with CSF concentrations of the axonal protein GFAB ${ }^{85}$. 
Antibodies against MOG can induce or contribute to demyelination in experimental autoimmune encephalomyelitis, the most commonly used experimental model of $\mathrm{MS}^{86}$. Furthermore, a neurological condition with pathological features similar to ADEM spontaneously develops in rodents and primates that undergo active immunization with MOG and in transgenic mice that have MOG-specific T cells and B cells ${ }^{87}$. Neuropathological examination of lesional brain biopsy samples from eight adults who were positive for MOG antibodies revealed extensive similarities to the pathology of $\mathrm{EAE}^{88,89}$.

Some studies have provided insight into the pathogenic mechanisms of MOG antibodies. In cell cultures, incubation with purified IgG from MOG antibody-positive patients led to loss of the microtubule cytoskeleton of oligodendrocytes ${ }^{90}$, and injection of purified IgG into the brains of mice resulted in myelin changes and altered expression of axonal proteins in the absence of inflammation, axonal loss and neuronal or astrocyte death ${ }^{91}$. This evidence suggests that MOG antibodies result in an autoimmune oligodendrogliopathy ${ }^{92}$.

The downstream effects of antibodies against extracellular receptors are varied, including internalization of the receptor, destruction of the antibody-antigen complex, and activation of complement ${ }^{81}$; These mechanisms can occur in isolation or in combination. Understanding the exact pathogenic mechanism of a given antibody can help when making treatment decisions. For example, anti-NMDA receptor antibodies cause internalization of the receptor $^{93}$, thereby causing selective, reversible decreases of NMDA receptor surface density, synaptic localization and currents ${ }^{93,94}$. Application of CSF from a patient with anti-NMDA receptor antibodies to brain tissue slice preparations is associated with reduced NMDA receptor density, and this effect is absent when anti-NMDA receptor antibodies are cleared from the $\mathrm{CSF}^{95}$. Of note, autopsy studies of patients with anti-NMDA receptor encephalitis reveal little inflammation, possibly because relatively little neuronal damage is caused by internalization of antibody-antigen complexes ${ }^{96}$. Strategies that reduce circulating antibodies, such as plasma exchange or $\mathrm{B}$ cell depletion, have considerable therapeutic efficacy ${ }^{97}$ : removal or reduction of circulating anti-NMDA receptor antibodies is thought to permit neuronal recovery of NMDA receptor complexes with relative preservation of the neurons ${ }^{93}$. However, hippocampal atrophy can occur, at least in adults ${ }^{97}$, suggesting that not all neurons recover completely. A postmortem study of adult patients with anti-NMDA receptor encephalitis revealed pronounced brain atrophy, although absent or reversible atrophy was observed in patients who recovered ${ }^{98}$, suggesting that disease severity is associated with cell survival.

Antibodies against the voltage-gated potassium channel (VGKC) complex require particular consideration in children. In adults, most anti-VGKC complex antibodies bind 
specifically to leucine-rich, glioma inactivated 1 (LGI-1) or contactin associated protein 2 (Caspr2), and are typically associated with limbic encephalitis with faciobrachiodystonic seizures (LGI-1 antibodies) or neuromyotonia with or without limbic encephalitis (Caspr2 antibodies). In children, most VGKC complex antibodies do not bind to LGI-1 or Caspr2 ${ }^{1}$. In a study of 39 children who were positive for anti-VGKC complex antibodies, $25 \%$ had antibodies against intracellular proteins within the complex. Furthermore, the clinical symptoms were highly variable, the presence of anti-VGKC antibodies did not correlate with the severity of neurological impairment, and treatment with immunosuppressive therapy was not clearly beneficial ${ }^{99}$. On the basis of these findings, detection of anti-VGKC complex antibodies in children is of limited diagnostic value.

\section{[H3] Intracellular antigens}

Neuroimmune disorders associated with antibodies against intracellular targets are rare in children. Such antibodies include those against Anti-neuronal nuclear antibody 1 (ANNA-1; also known as $\mathrm{Hu}$ ); purkinje cell cytoplasmic antibody (PCA-1; also known as Yo), anti-neuronal nuclear antibody 2 (ANNA-2; also known as Ri), Ma2, collapsing response mediator protein 5 (CRMP5; also known as Cv2), amphiphysin, Sox1, Sox2 and GAD-65. The pathogenicity of these antibodies differs greatly from that of antibodies against extracellular targets. These antibodies have only been identified in children in isolated case reports; in adults, they are often paraneoplastic and are associated with a range of central and peripheral symptoms ${ }^{100}$.

Anti-GAD-65 antibodies can be paraneoplastic, but are frequently associated with the non-paraneoplastic Stiff person syndrome ${ }^{101}$ and are also present in most patients with type 1 diabetes mellitus and in $\sim 4 \%$ of the general population. As such, the presence of GAD-65 antibodies at low titres in the absence of episodic hypertonicity should not be considered sufficient evidence of an autoimmune disorder. This point is particularly important when investigating a child with severe epilepsy: in such children, the presence of GAD-65 antibodies should not be viewed as definitive evidence of an neuroimmune disorder unless the antibody titre in the CSF is high ${ }^{102}$.

\section{[H1] Investigation of pathobiology}

\section{[H2] Marker assays}

Methods for the detection of disease-associated antibodies in samples from humans vary. For cytosolic or nuclear antigens, indirect immunohistochemistry followed by line blots or western 
blots is commonly used. For membrane protein antigens, cell-based assays, primary cultures, radioactive or fluorescence-based immunoprecipitation assays, enzyme-linked immunosorbent assay (ELISA) and indirect immunohistochemistry have all been used and have variable sensitivity and specificity. Solid-phase assays (such as western blotting and ELISA) are the most amenable to standardization and, therefore, broader use, but the results of these assays can be confounded by conformational changes in the antigens in the solid phase, which in turn can alter antibody binding. Cell-based assays, in which the antibody binds to the antigen in its physiological form, reduce this risk of antigen alterations. These cell-based assays are currently the gold standard for the detection of antibodies against cell-surface antigens, such as the NMDA receptor, AQP4 and MOG; positive results from these assays are more likely than positive results from other assays to indicate the presence of disease-associated antibodies.

The importance of assay choice cannot be overstated. In a direct, rigorous comparison of assays used to analyse 60 samples from adults with NMOSD, anti-AQP4 antibodies were detected in $48 \%$ of patients with tissue immunofluorescence, $60 \%$ with ELISA and $68 \%$ with cell-based flow cytometry ${ }^{103}$. In a comparison of assays for the detection of MOG antibodies, testing specifically for IgG1 antibodies substantially improved the specificity ${ }^{104}$. An alternative acknowledged method to prevent cross reactivity with IgM antibodies and hence improve the specificity ${ }^{105}$ is to use a secondary antibody against the Fc fragment of IgG.

The time point at which an assay is performed is also an important factor in its accuracy and value. Treatment with plasma exchange or IVIg will affect the accuracy of antibody measurements, particularly when the serum is tested. Antibody responses can also fluctuate during the course of illness: titres differ between periods of clinically active disease, periods of clinical remission, periods of treatment, and times of clinical symptom resurgence ${ }^{106}$. The presence of antibodies can also predede the first clinical manifestations, as demonstrated by the detection of AQP4 antibodies in patients who were being assessed for myasthenia gravis and did not develop symptoms of NMOSD for $>10$ years ${ }^{107}$.

\section{[H2] Animal models}

A major challenge in research into neuroimmune disorders is the absence of naturally-occurring animal models that recapitulate the human conditions. Animal models of CNS inflammation are generally induced by administration of an exogenous antigen, augmented by use of adjuvants and toxins to improve CNS access for peripherally-activated immune cells. These models are valuable for evaluation of specific disease aspects, but do not provide insight into the inciting biology in humans, and do not always predict treatment responses in patients. For disorders 
with a well-defined genetic contribution, typified by interferonopathies ${ }^{108}$, animals can be engineered to address specific aspects of disease pathogenesis, enabling targeted therapies to be developed.

\section{[H1] Genetics of neuroimmune disorders}

Several neuroimmune disorders have varying degress of genetic contributions that must be taken into account when managing a patient with one of these disorders. Clinical heterogeneity and variation in disease progression are widely recognized characteristics of MS and are thought to result from a combination of genetic and environmental risk factors ${ }^{109}$. Genome-wide association studies have identified many SNPs that are associated with $\mathrm{MS}^{110}$, but the strongest known individual genetic risk factor for paediatric and adult MS is human leukocyte antigen (HLA)DRB1*15:01, a class II allele within the major histocompatibility complex (MHC) on chromosome 6p21. In individuals of African descent, HLA-DRB1*15:03 is also associated with MS risk. Evidence for an association between anti-LGI-1 encephalitis and the MHC class II haplotype HLA-DR7 and HLA-DRB4 ${ }^{111,112}$ suggests that HLA alleles can also explain some susceptibility to other CNS autoimmune disorders, such as anti-NMDA receptor antibody encephalitis.

Most individuals with neuroimmune disorders are unlikely to have specific monogenic mutations, but studies of patients who do have these genetic mutations could provide insight into pathobiological pathways that offer new avenues for understanding neuroimmune conditions and treating them. Also noteworthy is that adrenoleukodystrophy and other genetic leukodystrophies are associated with clear immune responses to abnormal white matter. Immunosuppression has not been beneficial in these conditions ${ }^{113}$, suggesting that the immune response is secondary to the underlying disease biology and that the genetic mutation does not directly influence the behaviour of the immune system. Typical examples of monogenic disorder that result in CNS inflammation are influenza-mediated acute necrotizing encephalopathy, which is associated with autosomal dominant missense mutations in the heat shock protein RANBP2 ${ }^{114}$, and a predisposition to HSVE in people with inborn errors of anti-viral interferon immunity, such as mutations in Toll-like receptor 3 and interferon regulatory factor $3^{115,116}$.

Individuals can also have a genetic predisposition to macrophage activation in response to environmental triggers, a phenomenon called haemophagocytic lymphohistiocytosis. Various genetic abnormalities have been associated with this predisposition, including deficiency of the gene that encodes $X$-linked inhibitor of apoptosis $(X I A P)^{117}$ and mutations in PRF1, UNC13D (also known as MUNC13-4), STX11 and STXBP2. ${ }^{118}$ Neonatal-onset multisystem inflammatory disease caused by mutations in CIAS1, which encodes a protein responsible for cytokine 
activation ${ }^{119}$, is an autoinflammatory syndrome that presents with fever, chronic neutrophilic aseptic meningitis, uveitis, sensorineural hearing loss, urticarial skin rash and arthropathy.

Genetic mutations can also cause infection-independent inflammatory conditions through direct activation of inflammatory pathways. For example, AGS is a Mendelian disorder ${ }^{120-124}$ that presents with recurrent episodes of irritability and fever that is often accompanied by sterile CSF pleocytosis and considerable leukoencephalopathy with intracranial calcifications. A predisposition to secondary autoantibody formation can further contribute to disease pathogenesis ${ }^{125,126}$.

\section{[H1] Research priorities}

Collaborative networks and the use of shared clinical evaluative platforms, standardized biological assays, structured neuroimaging protocols and partnered research programs are increasingly enabling advances in clinical care and research in neuroimmune disorders. However, further improvements through the development of targeted therapies and precision medicine will depend on collaborative research in several key areas. Below, we set out four areas of research that we consider to be priorities for advancing knowledge in a way that will improve clinical care.

\section{[H2] Collection of core clinical data}

Rigorous and consistent collection of core clinical data will harmonize records and streamline research. Consistent evaluation of patient outcomes is essential; for this evaluation, expert working groups can choose from instruments such as the NIH Toolbox, the Patient-Reported Outcomes Measurement Information System (PROMIS), and the Quality of Life in Neurological Disorders (Neuro-QOL) system. However, the suitability of such tools for paediatric patients needs to be considered, as normative data for many of these tests might not be available owing to difficulties with obtaining unbiased data from healthy children of different ages, and the

effects of neuroimmune disorders on maturing neural networks might be expected to differ from the effects on older brains. Furthermore, such effects might not be detectable immediately and might become more apparent as the child ages and abnormal development of pathways injured during the acute phase of their illness becomes apparent. Use of serial imaging will determine whether brain growth trajectories in children who have neuroimmune disorders are as expected for their age.

\section{[H2] Cooperative investigation of treatment}


Success of the cooperative care models in childhood oncology is a testament to the value of collaborative treatment plans. Although phase III clinical trials remain the gold standard for evidence-based treatment, such studies are not feasible in rare diseases owing to a lack of statistical power. Furthermore, when biologically plausible mechanisms for treatment are discovered for fatal disorders, the impetus to bring such therapies promptly to the children who need them is obviously strong. Collaborative agreement regarding treatment algorithms and consistent diagnostic evaluation, and consensus regarding longitudinal observations to establish outcomes and ensure safety would advance care in circumstances where phase III trials are not possible.

\section{[H2] Development of biomarker assays}

Development of biological assays for markers of neuroimmune disorders will advance the field, and there are several fundamental requirements for this work. Validation of assays requires the ability to compare the sensitivity and specificity across different neuroimmune conditions and against non-immune CNS diseases; establishing shared, well-characterized biorepositories will be essential for future assay development. Assays can also become refined over time; for example, the use of cell-based assays has improved the specificity for detection of AQP4 antibodies $^{127}$, and specific tests for the $\lg G$ subclass of MOG antibodies has improved detection ${ }^{49}$. Such refinement and the defining of gold standard assays can be achieved through multi-lab comparisons of shared samples, as has proved effective in the case of AQP4 antibody assays $^{128,129}$.

\section{[H2] Genetic studies of neuroimmune disorders}

Further studies to identify heritable immune disorders and genetic modifiers of disease are also necessary. Whole-exome sequencing and whole-genome sequencing are emerging as approaches that could identifiy novel genetic contributions to neuroimmune diseases. In principle, identification of disruption to a specific genetically mediated immune pathway could result in development of targeted treatment ${ }^{130}$. Furthermore, next-generation sequencing of CSF or brain biopsy tissue could identify novel or unsuspected pathogens that are either aetiological agents or key immune triggers.

\section{[H1] Conclusions}

Advances have been made in the clinical care of patients with neuroimmune disorders, but treatment could be improved by greater knowledge about the pathobiological mechanisms that 
cause these conditions and by more collaborative clinical data recording. The above research priorities are highlighted with the goal of galvanizing the community of clinicians and researchers working in the field of neuroimmune disorders towards a shared vision of moreprecise, targeted therapeutics. B-cell-depleting therapies, antibody therapies that target specific antigens on distinct immune cell subsets, and modulation of the type 1 interferon pathway in AGS and other interferonopathies are examples of exciting advances that hold great promise, but advances in the research areas discussed will be required for if such promise is to be realized.

\section{Review criteria}

References for this Review were identified through searches of PubMed by use of the search terms "autoimmune", "inflammatory", "p(a)ediatrics”, "N-methyl-D-aspartate receptor", "MOG”, "AQP4", "Aquaporin", "neuromyelitis", "transverse myelitis" and "optic neuritis". Papers published from 1990 to January 2018 were included. Only papers published in English were reviewed. The final reference list was generated on the basis of relevance to the topics covered in this review. 


\section{Competing interests}

B.B. is a co-inventor on several NIH patents related to daclizumab therapy and, as such, has received patent royalty payments from $\mathrm{NIH}$.

W.D.G. receives grant support from the NIH, NSF, PCORI, American Epilepsy Society, Epilepsy Foundation, CURE and the Infantile Epilepsy Research Foundation, sits on the editorial board of Epilepsia, holds stock from Johnson and Johnson, GlaxoSmithKline, Eli Lilly, Pfizer, Sieman, and General Electrics.

R.C.D. has received research funding from the National Health and Medical Research Council, MS Research Australia, Star Scientific Foundation, Pfizer Neuroscience, Tourette Syndrome Association, University of Sydney, and the Petre Foundation. He has also received honoraria from Biogen and Bristol-Myers Squibb as an invited speaker.

R.T. has received funding from the NIH R21-NS084264 and U01-NS081041.

E.A.Y. has received funding from NMSS, the MS Society of Canada, the Rare Disease Foundation, CIHI/CMSMS, Mario Batalli Foundation, PCORI, CIHR, SickKids Innovation Fund, SickKids Foundation. She also is an ACI consultant, EXCEMED (speaker's honoraria), travel support and/or honoraria Jamacian Pediatrics Association, Saudi Arabian Child Neurology Association, Guthy Jackson Foundation and the Nicholas Foundation.

S.P> and the Mayo Clinic have a financial interest in patents (\#12/678,350 filed 2010 and \#12/573,942 filed 2008) that relate to functional AQP4 IgG and NMO IgG assays and NMO IgG as a cancer marker S.P. has consulted for Alexion Pharmaceuticals, Medimmune and Chugai Pharma but has received no personal fees or personal compensation for these consulting activities; all compensation for consulting activities is paid directly to Mayo Clinic. S.P. has also received a research grant from Alexion pharmaceuticals for an investigator-initiated study, as well as support from the NIH (RO1 NS065829-01) and the Guthy Jackson Charitable Foundation for NMO research.

B.G. serves on the medical and scientific advisory board of the Transverse Myelitis Association. He has received consulting income from EMD Serono and Novartis. He has grant support from Medimmune, Biogen, Chugai, Acorda, PCORI, NIH and the Guthy Jackson Foundation. G.M. has received grant support from $\mathrm{SOBI}$, Novartis, Regeneron and Lilly. C.A.P. serves on the medical and scientific advisory board of the Transverse Myelitis Association.

B.A. is funded by NIH [R01 NR012907, R01 NR014449, R01 NR014449, R01 NR015738]. A.W. has received research/grant support from Medimmune, Chugai and NIHthe National Institutes of Health (K23NS069806, R01NS071463), National Multiple Sclerosis Society, 
Foerderer Award (Children's Hospital of Philadelphia), Biogen Idec, IONIS Pharmaceutics, Novartis Pharmaceutical Corporation, Elise's Corner (Akron Community Foundation), The Calliope Joy Foundation, Lynn Saligman League, speaker's fee from SUNY Downstate Medical Center, and receives publishing royalties from UpToDate Inc.

All other authors declare no competing interests.

\section{Author contributions}

E.W., Y.H., A.V. and B.B. drafted the manuscript. All authors participated in manuscript development and review.

\section{Acknowledgements}

This reviewed was derived from a meeting of clinician and scientists as well as patient advocacy groups which was held in Washington DC in June 2014 funded by the Ikaria Fund at Children's National Health System. 
Figure 1 | Diagnostic algorithm for for a first presentation of acquired demyelinating syndrome (ADS) in a child without encephalopathy. When clinical and radiologoical features suggestive of neuromyelitis optica spectrum disorder are seen, AQP4-Ab should be tested. If the clinical presentation is suggestive of multiple sclerosis (MS) the 2017 diagnostic criteria should be applied. The presence of intrathecal oligoclonal band can be used for dissemination in time criteria. If non-MS and AQP4-Ab negative MOG-Ab asspciated disease should be considered. If no diagnosis after the first ADS MRI and MOG and AQP4 antibodies should be retested at time of clinical relapse.

Figure 2| Diagnostic algorithm for patients with neuroimmune disorders presenting with encephalopathy. For patienst presenting with sever encephalopathy with movement disorder/+ seizures/+ disautonomia in keeping with the phenotype described in AntiNMDAR encephalitis, NMDAR-Ab should be tested. For all other children, exclusion of infective ethiology should be performed, this can be done in parallal to the autoinflammatory work-up. Normal MRI is frequently seen in children with Ant-NMDAR encephalitis. Children with Anti-NMDAR encephalitis presenting following HSV encephalitis typically present with blateral asymmetrical leukoencephalopathy. Other MRI pattern can aid in diagnosis. Of note most children relapsing following ADEM will have MOG-Ab.

[Au: Figure 3 as supplied is too small for print. For maximum print quality, please provide the original images (or high-quality images) of each panel separately without any text of labels on. However, please also provide versions with arrows that indicate the features mentioned in the legend so that the reader is clear about which elements in the images correspond to which features. We will add these arrows to the unlabelled versions in our journal style.] Figure 3 | Imaging features of neuroimmune conditions. a | Typical MRI images from a child with persistent paraesthesias. Fluid-attenuated inversion recovery (FLAIR) images show juxtacortical and periventricular lesions (left) and an enhancing lesion (right). [Au: I suggest that A and B are included in a single panel (a) and referred to as left and right for clarity in the legend.] These features satisfy the 2010 McDonald criteria for MS. c | An axial MRI scan shows a T2 hyperintensity and patchy contrast enhancement of the right insula, inferior frontal lobe, and anterior temporal lobe in a child with post-herpes simplex virus antiNMDA receptor encephalitis. $d \mid$ An axial FLAIR image that shows multifocal lesions in the cortex in a 17-year-old with biopsy-proven small vessel vasculitis of the CNS. e | A sagittal T2- 
weighted image of the brain of a child with NMOSD, showing a cervical lesion that extends for approximately three vertebral segments. $f$ | [Au: What type of image is this and what does it show?] A child who has an area postrema syndrome and is positive for anti-AQP4 antibodies, thereby meeting the diagnostic criteria for NMOSD. [Au: Changes to wording OK? This sentence was referenced to part e, but part e was already referred to, so I assume this reference refers to part f. Please check.] g | [Au: Please check that this refers to the right panel.] An MRI scan of the cervical spine in a child with acute limb weakness. The image revealed bilateral lesions restricted to the grey matter, consistent with acute flaccid myelitis. $\mathrm{h}$ | Anti-MOG antibody associated disease that presents with acute disseminated encephalomyelitis (ADEM). [Au: What type of image is this, and what are the features that it actually shows?] i | A relapse 4 months after ADEM with encephalopathy and ataxia. [Au: What type of image is this, and what are the features it actually shows?]

[Au: Please provide a list of up to 6 brief bullet points, each no more than 2 sentences long, highlighting the take-home messages of the Review.]

1. CNS autoimmunity resulted in a paradigm shift in the diagnostic approach of children with neurological disorders

2. Specific clinic-iimunolopgical syndromes have been reported secondary to pathogenic autoantibodies but a proportion of children with a presume neuroimmune disorder are "seronegative"

3. The sensitivity and specificity of the different assays requires strict clinical inclusion criteria in order to determine the applicability of the test in a clinical setting. It is important to distinguish technically false positivity from a biological valid seropositive result. The current "gold standard" remains the clinical presentation.

4. The outcome of children treated with immunotherapies appears to be superior

5. Current therapeutic strategies are largely centre-specific and consensus guidelines are yet to be formulated

6. Understanding of pathobiological mechanisms may help in the development of new targeted treatments

7. Inctions exposure and potential genetics influence remained to be discovered

[Au: For references that are particularly worth reading (5-10\% of the total), please provide a single bold sentence that indicates the significance of the work. 
1. Armangue T, Petit-Pedrol M, Dalmau J. Autoimmune encephalitis in children. J Child Neurol 2012;2012/09/01:1460-1469.

2. Waldman A, Ghezzi A, Bar-Or A, Mikaeloff Y, Tardieu M, Banwell B. Multiple sclerosis in children: an update on clinical diagnosis, therapeutic strategies, and research. Lancet Neurol2014: 936-948.

3. Graus F, Titulaer MJ, Balu R, et al. A clinical approach to diagnosis of autoimmune encephalitis. Lancet Neurol 2016;15:391-404.

4. Hacohen Y, Vincent A. Autoimmune neurological disorders-does the age matter? Eur J Paediatr Neurol 2018;22:341-343.

5. Titulaer MJ, Hoftberger R, lizuka T, et al. Overlapping demyelinating syndromes and anti-NMDA receptor encephalitis. Ann Neurol 2014.

6. Hacohen $Y$, Absoud $M$, Hemingway $C$, et al. NMDA receptor antibodies associated with distinct white matter syndromes. Neurol Neuroimmunol Neuroinflamm 2014;1:e2.

7. Verhey LH, Branson HM, Laughlin S, et al. Development of a standardized MRI scoring tool for CNS demyelination in children. AJNR Am J Neuroradiol 2013;34:1271-1277.

8. Florance NR, Davis RL, Lam C, et al. Anti-N-methyl-D-aspartate receptor (NMDAR) encephalitis in children and adolescents. Ann Neurol 2009;66:11-18.

9. Polman CH, Reingold SC, Banwell B, et al. Diagnostic criteria for multiple sclerosis: 2010 revisions to the McDonald criteria. Ann Neurol 2011;69:292-302.

10. Kim HJ, Paul F, Lana-Peixoto MA, et al. MRI characteristics of neuromyelitis optica spectrum disorder: an international update. Neurology 2015;84:1165-1173.

11. Aliaga ES, Barkhof F. MRI mimics of multiple sclerosis. Handb Clin Neurol 2014;122:291-316.

12. Absinta M, Vuolo L, Rao A, et al. Gadolinium-based MRI characterization of leptomeningeal inflammation in multiple sclerosis. Neurology 2015;85:18-28.

13. Manogaran $\mathrm{P}$, Vavasour I, Borich $\mathrm{M}$, et al. Corticospinal tract integrity measured using transcranial magnetic stimulation and magnetic resonance imaging in neuromyelitis optica and multiple sclerosis. Multiple sclerosis (Houndmills, Basingstoke, England) 2016;22:43-50.

14. Newey CR, Sarwal A, Hantus S. [18F]-Fluoro-Deoxy-Glucose Positron Emission Tomography Scan Should Be Obtained Early in Cases of Autoimmune Encephalitis. Autoimmune Diseases 2016;2016:6.

15. Herranz E, Gianni C, Louapre C, et al. Neuroinflammatory component of gray matter pathology in multiple sclerosis. Ann Neurol 2016;80:776-790.

16. Hacohen Y, Rossor T, Mankad K, et al. 'Leukodystrophy-like' phenotype in children with myelin oligodendrocyte glycoprotein antibody-associated disease. Dev Med Child Neurol 2017.

17. Rodero MP, Crow YJ. Type I interferon-mediated monogenic autoinflammation: The type I interferonopathies, a conceptual overview. J Exp Med 2016;213:2527-2538.

18. Varadkar S, Bien CG, Kruse CA, et al. Rasmussen's encephalitis: clinical features, pathobiology, and treatment advances. Lancet Neurol 2014;13:195-205.

19. Han S, Lin YC, Wu T, et al. Comprehensive Immunophenotyping of Cerebrospinal Fluid Cells in Patients with Neuroimmunological Diseases. Journal of immunology 2014.

20. Edwards KR, Goyal J, Plavina T, et al. Feasibility of the use of combinatorial chemokine arrays to study blood and CSF in multiple sclerosis. PloS one 2013;8:e81007.

21. von Budingen HC, Kuo TC, Sirota M, et al. B cell exchange across the blood-brain barrier in multiple sclerosis. J Clin Invest 2012;122:4533-4543.

22. Dale RC, Brilot F. Biomarkers of inflammatory and auto-immune central nervous system disorders. Curr Opin Pediatr 2010;22:718-725.

23. Rice $\mathrm{GI}$, Forte $\mathrm{GM}$, Szynkiewicz $\mathrm{M}$, et al. Assessment of interferon-related biomarkers in AicardiGoutieres syndrome associated with mutations in TREX1, RNASEH2A, RNASEH2B, RNASEH2C, SAMHD1, and ADAR: a case-control study. Lancet Neurol 2013;12:1159-1169. 
24. Yokobori S, Hosein K, Burks S, Sharma I, Gajavelli S, Bullock R. Biomarkers for the clinical differential diagnosis in traumatic brain injury--a systematic review. CNS Neurosci Ther 2013;19:556565.

25. Titulaer MJ, McCracken L, Gabilondo I, et al. Treatment and prognostic factors for long-term outcome in patients with anti-NMDA receptor encephalitis: an observational cohort study. Lancet Neurol 2013;12:157-165.

26. Blackman JA, Patrick PD, Buck ML, Rust RS, Jr. Paroxysmal autonomic instability with dystonia after brain injury. Archives of neurology 2004;61:321-328.

27. Mohammad SS, Jones $H$, Hong $M$, et al. Symptomatic treatment of children with anti-NMDAR encephalitis. Dev Med Child Neurol 2016;58:376-384.

28. Wright S, Vincent A. Pediatric Autoimmune Epileptic Encephalopathies. J Child Neurol 2017;32:418-428.

29. Schmitt SE, Pargeon K, Frechette ES, Hirsch L, Dalmau J, Friedman D. Extreme delta brush: a unique EEG pattern in adults with anti-NMDA receptor encephalitis. Neurology 2012;79:1094-1100.

30. Dale RC, Gorman MP, Lim M. Autoimmune encephalitis in children: clinical phenomenology, therapeutics, and emerging challenges. Curr Opin Neurol 2017;30:334-344.

31. Dalakas MC. Mechanistic effects of IVIg in neuroinflammatory diseases: conclusions based on clinicopathologic correlations. J Clin Immunol 2014;34 Suppl 1:S120-126.

32. Toledano M, Pittock SJ. Autoimmune Epilepsy. Semin Neurol 2015;35:245-258.

33. Wolf NI, Toro C, Kister I, et al. DARS-associated leukoencephalopathy can mimic a steroidresponsive neuroinflammatory disorder. Neurology 2015;84:226-230.

34. Schaad UB, Lips U, Gnehm HE, Blumberg A, Heinzer I, Wedgwood J. Dexamethasone therapy for bacterial meningitis in children. Swiss Meningitis Study Group. Lancet 1993;342:457-461.

35. Prasad K, Singh MB, Ryan $\mathrm{H}$. Corticosteroids for managing tuberculous meningitis. Cochrane Database Syst Rev 2016;4:CD002244.

36. Turner MT, Nayak S, Kuhn M, Roehm PC. The Effects of Dexamethasone and Acyclovir on a Cell Culture Model of Delayed Facial Palsy. Otol Neurotol 2014;35:712-718.

37. Nosadini M, Mohammad SS, Corazza F, et al. Herpes simplex virus-induced anti-N-methyl-daspartate receptor encephalitis: a systematic literature review with analysis of 43 cases. Dev Med Child Neurol 2017;59:796-805.

38. Krupp LB, Tardieu M, Amato MP, et al. International Pediatric Multiple Sclerosis Study Group criteria for pediatric multiple sclerosis and immune-mediated central nervous system demyelinating disorders: revisions to the 2007 definitions. Mult Scler 2013;19:1261-1267.

39. Dale RC, Brilot F, Duffy LV, et al. Utility and safety of rituximab in pediatric autoimmune and inflammatory CNS disease. Neurology 2014;83:142-150.

40. Hacohen $\mathrm{Y}$, Wong YY, Lechner $\mathrm{C}$, et al. Disease Course and Treatment Responses in Children With Relapsing Myelin Oligodendrocyte Glycoprotein Antibody-Associated Disease. JAMA Neurol 2018.

41. Raftopoulos R, Hickman SJ, Toosy A, et al. Phenytoin for neuroprotection in patients with acute optic neuritis: a randomised, placebo-controlled, phase 2 trial. Lancet Neurol 2016;15:259-269.

42. Suhs KW, Hein K, Sattler MB, et al. A randomized, double-blind, phase 2 study of erythropoietin in optic neuritis. Ann Neurol 2012;72:199-210.

43. Agarwal S, Keller JR, Nunneley CE, et al. Therapeutic Plasma Exchange Use in Pediatric Neurologic Disorders at a Tertiary Care Center: A 10-Year Review. J Child Neurol 2018;33:140-145.

44. Aljebab F, Choonara I, Conroy S. Systematic Review of the Toxicity of Long-Course Oral Corticosteroids in Children. PLoS One 2017;12:e0170259.

45. Hauser SL, Bar-Or A, Comi G, et al. Ocrelizumab versus Interferon Beta-1a in Relapsing Multiple Sclerosis. New England Journal of Medicine 2017;376:221-234. 
46. Nosadini M, Alper G, Riney CJ, et al. Rituximab monitoring and redosing in pediatric neuromyelitis optica spectrum disorder. Neurol Neuroimmunol Neuroinflamm 2016;3:e188.

47. Goldbach-Mansky R. Immunology in clinic review series; focus on autoinflammatory diseases: update on monogenic autoinflammatory diseases: the role of interleukin (IL)-1 and an emerging role for cytokines beyond IL-1. Clin Exp Immunol 2012;167:391-404.

48. Araki M, Matsuoka T, Miyamoto K, et al. Efficacy of the anti-IL-6 receptor antibody tocilizumab in neuromyelitis optica: a pilot study. Neurology 2014;82:1302-1306.

49. Hacohen $\mathrm{Y}, \mathrm{Absoud} \mathrm{M}$, Deiva K, et al. Myelin oligodendrocyte glycoprotein antibodies are associated with a non-MS course in children. Neurol Neuroimmunol Neuroinflamm 2015;2:e81.

50. Ketelslegers IA, Van Pelt DE, Bryde S, et al. Anti-MOG antibodies plead against MS diagnosis in an Acquired Demyelinating Syndromes cohort. Mult Scler 2015.

51. Hacohen Y, Mankad K, Chong WK, et al. Diagnostic algorithm for relapsing acquired demyelinating syndromes in children. Neurology 2017.

52. Gresa-Arribas N, Titulaer MJ, Torrents A, et al. Antibody titres at diagnosis and during follow-up of anti-NMDA receptor encephalitis: a retrospective study. The Lancet Neurology 2014;13:167-177.

53. Gresa-Arribas N, Titulaer MJ, Torrents A, et al. Antibody titres at diagnosis and during follow-up of anti-NMDA receptor encephalitis: a retrospective study. The Lancet Neurology 2014;13:167-177.

54. Hennes EM, Baumann M, Schanda K, et al. Prognostic relevance of MOG antibodies in children with an acquired demyelinating syndrome. Neurology 2017.

55. Wingerchuk DM, Banwell B, Bennett JL, et al. International consensus diagnostic criteria for neuromyelitis optica spectrum disorders. Neurology 2015.

56. Duignan S, Wright S, Rossor T, et al. Myelin oligodendrocyte glycoprotein and aquaporin-4 antibodies are highly specific in children with acquired demyelinating syndromes. Dev Med Child Neurol 2018.

57. Quintana FJ, Patel B, Yeste A, et al. Epitope spreading as an early pathogenic event in pediatric multiple sclerosis. Neurology 2014;83:2219-2226.

58. Schwarz A, Balint B, Korporal-Kuhnke M, et al. B-cell populations discriminate between pediatric- and adult-onset multiple sclerosis. Neurol Neuroimmunol Neuroinflamm 2017;4:e309.

59. Dhaunchak AS, Becker C, Schulman H, et al. Implication of perturbed axoglial apparatus in early pediatric multiple sclerosis. Ann Neurol 2012;71:601-613.

60. Bar-Or A, Hintzen RQ, Dale RC, Rostasy K, Bruck W, Chitnis T. Immunopathophysiology of pediatric CNS inflammatory demyelinating diseases. Neurology 2016;87:S12-19.

61. Pillai SC, Hacohen Y, Tantsis E, et al. Infectious and Autoantibody-Associated Encephalitis: Clinical Features and Long-term Outcome. Pediatrics 2015.

62. Hacohen $\mathrm{Y}$, Wright $\mathrm{S}$, Waters $\mathrm{P}$, et al. Paediatric autoimmune encephalopathies: clinical features, laboratory investigations and outcomes in patients with or without antibodies to known central nervous system autoantigens. J Neurol Neurosurg Psychiatry 2013;84:748-755.

63. Hacohen $Y$, Nishimoto $Y$, Fukami $Y$, et al. Paediatric brainstem encephalitis associated with glial and neuronal autoantibodies. Dev Med Child Neurol 2016;58:836-841.

64. Cellucci T, Benseler SM. Diagnosing central nervous system vasculitis in children. Curr Opin Pediatr 2010;22:731-738.

65. Twilt M, Benseler SM. CNS vasculitis in children. Mult Scler Relat Disord 2013;2:162-171.

66. Elbers J, Halliday W, Hawkins C, Hutchinson C, Benseler SM. Brain biopsy in children with primary small-vessel central nervous system vasculitis. Ann Neurol 2010;68:602-610.

67. Gable MS, Sheriff H, Dalmau J, Tilley DH, Glaser CA. The frequency of autoimmune N-methyl-Daspartate receptor encephalitis surpasses that of individual viral etiologies in young individuals enrolled in the California Encephalitis Project. Clin Infect Dis 2012;54:899-904. 
68. Armangue T, Leypoldt F, Dalmau J. Autoimmune encephalitis as differential diagnosis of infectious encephalitis. Curr Opin Neurol 2014;27:361-368.

69. Bamford A, Crowe BH, Hacohen Y, et al. Pediatric Herpes Simplex Virus Encephalitis Complicated by N-Methyl-D-aspartate Receptor Antibody Encephalitis. J Pediatric Infect Dis Soc 2015;4:e17-21.

70. Hacohen $\mathrm{Y}$, Deiva K, Pettingill P, et al. N-methyl-D-aspartate receptor antibodies in post-herpes simplex virus encephalitis neurological relapse. Mov Disord 2014;29:90-96.

71. Schein F, Gagneux-Brunon A, Antoine JC, et al. Anti-N-methyl-D-aspartate receptor encephalitis after Herpes simplex virus-associated encephalitis: an emerging disease with diagnosis and therapeutic challenges. Infection 2016.

72. Armangue $\mathrm{T}$, Moris $\mathrm{G}$, Cantarin-Extremera $\mathrm{V}$, et al. Autoimmune post-herpes simplex encephalitis of adults and teenagers. Neurology 2015;85:1736-1743.

73. Banwell B, Krupp L, Kennedy J, et al. Clinical features and viral serologies in children with multiple sclerosis: a multinational observational study. The Lancet Neurology 2007;6:773-781.

74. Steiner I, Kennedy PG, Pachner AR. The neurotropic herpes viruses: herpes simplex and varicellazoster. Lancet Neurol 2007;6:1015-1028.

75. Alotaibi S, Kennedy J, Tellier R, Stephens D, Banwell B. Epstein-Barr virus in pediatric multiple sclerosis. Jama 2004;291:1875-1879.

76. Waubant E, Mowry EM, Krupp L, et al. Common viruses associated with lower pediatric multiple sclerosis risk. Neurology 2011;76:1989-1995.

77. Ramagopalan SV, Dobson R, Meier UC, Giovannoni G. Multiple sclerosis: risk factors, prodromes, and potential causal pathways. Lancet Neurol 2010;9:727-739.

78. Hoftberger R, Sepulveda M, Armangue T, et al. Antibodies to MOG and AQP4 in adults with neuromyelitis optica and suspected limited forms of the disease. Mult Scler 2014.

79. Abdul-Rahman ZM, Panegyres PK, Roeck M, et al. Anti-N-methyl-D-aspartate receptor encephalitis with an imaging-invisible ovarian teratoma: a case report. J Med Case Rep 2016;10:296.

80. Horellou P, Wang M, Keo V, et al. Increased interleukin-6 correlates with myelin oligodendrocyte glycoprotein antibodies in pediatric monophasic demyelinating diseases and multiple sclerosis. J Neuroimmunol 2015;289:1-7.

81. Dalmau J, Geis C, Graus F. Autoantibodies to Synaptic Receptors and Neuronal Cell Surface Proteins in Autoimmune Diseases of the Central Nervous System. Physiol Rev 2017;97:839-887.

82. Lim M, Hacohen Y, Vincent A. Autoimmune encephalopathies. Pediatr Clin North Am 2015;62:667-685.

83. Pittock SJ, Lucchinetti CF. Neuromyelitis optica and the evolving spectrum of autoimmune aquaporin-4 channelopathies: a decade later. Ann N Y Acad Sci 2015.

84. Papadopoulos MC, Verkman AS. Aquaporin water channels in the nervous system. Nat Rev Neurosci 2013;14:265-277.

85. Sato DK, Callegaro D, de Haidar Jorge FM, et al. Cerebrospinal fluid aquaporin-4 antibody levels in neuromyelitis optica attacks. Ann Neurol 2014;76:305-309.

86. Iglesias A, Bauer J, Litzenburger T, Schubart A, Linington C. T- and B-cell responses to myelin oligodendrocyte glycoprotein in experimental autoimmune encephalomyelitis and multiple sclerosis. Glia 2001;36:220-234.

87. Krishnamoorthy $\mathrm{G}$, Lassmann $\mathrm{H}$, Wekerle $\mathrm{H}$, Holz A. Spontaneous opticospinal encephalomyelitis in a double-transgenic mouse model of autoimmune T cell/B cell cooperation. J Clin Invest 2006;116:2385-2392.

88. Kortvelyessy P, Breu M, Pawlitzki M, et al. ADEM-like presentation, anti-MOG antibodies, and MS pathology: TWO case reports. Neurol Neuroimmunol Neuroinflamm 2017;4:e335.

89. Hoftberger R, Lassmann H. Inflammatory demyelinating diseases of the central nervous system. Handb Clin Neurol 2017;145:263-283. 
90. Dale RC, Tantsis EM, Merheb V, et al. Antibodies to MOG have a demyelination phenotype and affect oligodendrocyte cytoskeleton. Neurol Neuroimmunol Neuroinflamm 2014;1:e12.

91. Saadoun S, Waters P, Owens GP, Bennett JL, Vincent A, Papadopoulos MC. Neuromyelitis optica MOG-IgG causes reversible lesions in mouse brain. Acta Neuropathol Commun 2014;2:35.

92. Reindl M, Jarius S, Rostasy K, Berger T. Myelin oligodendrocyte glycoprotein antibodies: How clinically useful are they? Curr Opin Neurol 2017.

93. Moscato EH, Peng X, Jain A, Parsons TD, Dalmau J, Balice-Gordon RJ. Acute mechanisms underlying antibody effects in anti-NMDA receptor encephalitis. Ann Neurol 2014.

94. Mikasova L, De Rossi $P$, Bouchet $D$, et al. Disrupted surface cross-talk between NMDA and Ephrin-B2 receptors in anti-NMDA encephalitis. Brain : a journal of neurology 2012;135:1606-1621.

95. Planaguma J, Leypoldt F, Mannara F, et al. Human N-methyl D-aspartate receptor antibodies alter memory and behaviour in mice. Brain 2015;138:94-109.

96. Bien CG, Vincent A, Barnett MH, et al. Immunopathology of autoantibody-associated encephalitides: clues for pathogenesis. Brain 2012;135:1622-1638.

97. Finke C, Kopp UA, Pajkert A, et al. Structural Hippocampal Damage Following Anti-N-Methyl-DAspartate Receptor Encephalitis. Biol Psychiatry 2016;79:727-734.

98. Heine J, Pruss H, Bartsch T, Ploner CJ, Paul F, Finke C. Imaging of autoimmune encephalitis-Relevance for clinical practice and hippocampal function. Neuroscience 2015;309:68-83.

99. Hacohen $\mathrm{Y}$, Singh R, Rossi M, et al. Clinical relevance of voltage-gated potassium channelcomplex antibodies in children. Neurology 2015.

100. Graus F, Dalmau J. Paraneoplastic neurological syndromes. Curr Opin Neurol 2012;25:795-801.

101. Schiff D, Dalmau J, Myers DJ. Anti-GAD antibody positive stiff-limb syndrome in multiple myeloma. J Neurooncol 2003;65:173-175.

102. Fouka P, Alexopoulos H, Akrivou S, Trohatou O, Politis PK, Dalakas MC. GAD65 epitope mapping and search for novel autoantibodies in GAD-associated neurological disorders. J Neuroimmunol 2015;281:73-77.

103. Waters PJ, McKeon A, Leite MI, et al. Serologic diagnosis of NMO A multicenter comparison of aquaporin-4-IgG assays. Neurology 2012;78:665-671.

104. Waters P, Woodhall M, O'Connor KC, et al. MOG cell-based assay detects non-MS patients with inflammatory neurologic disease. Neurol Neuroimmunol Neuroinflamm 2015;2:e89.

105. Hennes EM, Baumann M, Schanda K, et al. Prognostic relevance of MOG antibodies in children with an acquired demyelinating syndrome. Neurology 2017;89:900-908.

106. Hansen HC, Klingbeil C, Dalmau J, Li W, Weissbrich B, Wandinger KP. Persistent intrathecal antibody synthesis 15 years after recovering from anti-N-methyl-D-aspartate receptor encephalitis. JAMA Neurol 2013;70:117-119.

107. Leite MI, Coutinho E, Lana-Peixoto M, et al. Myasthenia gravis and neuromyelitis optica spectrum disorder: a multicenter study of 16 patients. Neurology 2012;78:1601-1607.

108. Crow YJ, Manel N. Aicardi-Goutieres syndrome and the type I interferonopathies. Nat Rev Immunol 2015;15:429-440.

109. Waubant E, Ponsonby AL, Pugliatti M, Hanwell H, Mowry EM, Hintzen RQ. Environmental and genetic factors in pediatric inflammatory demyelinating diseases. Neurology 2016;87:S20-27.

110. International Multiple Sclerosis Genetics C, Wellcome Trust Case Control C, Sawcer S, et al. Genetic risk and a primary role for cell-mediated immune mechanisms in multiple sclerosis. Nature 2011;476:214-219.

111. Kim TJ, Lee ST, Moon J, et al. Anti-LGI1 encephalitis is associated with unique HLA subtypes. Annals of Neurology 2017;81:183-192.

112. van Sonderen A, Roelen DL, Stoop JA, et al. Anti-LGI1 encephalitis is strongly associated with HLA-DR7 and HLA-DRB4. Annals of Neurology 2017;81:193-198. 
113. Naidu S, Bresnan MJ, Griffin D, O'Toole S, Moser HW. Childhood adrenoleukodystrophy. Failure of intensive immunosuppression to arrest neurologic progression. Archives of neurology 1988;45:846848.

114. Neilson DE. The interplay of infection and genetics in acute necrotizing encephalopathy. Curr Opin Pediatr 2010;22:751-757.

115. Sancho-Shimizu V, Perez de Diego R, Jouanguy E, Zhang SY, Casanova JL. Inborn errors of antiviral interferon immunity in humans. Curr Opin Virol 2011;1:487-496.

116. Andersen LL, Mork N, Reinert LS, et al. Functional IRF3 deficiency in a patient with herpes simplex encephalitis. J Exp Med 2015;212:1371-1379.

117. Latour S, Aguilar C. XIAP deficiency syndrome in humans. Semin Cell Dev Biol 2015;39:115-123. 118. Sepulveda FE, de Saint Basile G. Hemophagocytic syndrome: primary forms and predisposing conditions. Curr Opin Immunol 2017;49:20-26.

119. Aksentijevich I, Nowak M, Mallah M, et al. De novo CIAS1 mutations, cytokine activation, and evidence for genetic heterogeneity in patients with neonatal-onset multisystem inflammatory disease (NOMID): a new member of the expanding family of pyrin-associated autoinflammatory diseases. Arthritis Rheum 2002;46:3340-3348.

120. Rice GI, Bond J, Asipu A, et al. Mutations involved in Aicardi-Goutieres syndrome implicate SAMHD1 as regulator of the innate immune response. Nat Genet 2009;41:829-832.

121. Rice GI, del Toro Duany Y, Jenkinson EM, et al. Gain-of-function mutations in IFIH1 cause a spectrum of human disease phenotypes associated with upregulated type I interferon signaling. Nat Genet 2014;46:503-509.

122. Rice GI, Kasher PR, Forte GM, et al. Mutations in ADAR1 cause Aicardi-Goutieres syndrome associated with a type I interferon signature. Nat Genet 2012;44:1243-1248.

123. Crow YJ, Hayward BE, Parmar R, et al. Mutations in the gene encoding the 3'-5' DNA exonuclease TREX1 cause Aicardi-Goutieres syndrome at the AGS1 locus. Nat Genet 2006;38:917-920.

124. Crow YJ, Leitch A, Hayward BE, et al. Mutations in genes encoding ribonuclease $\mathrm{H} 2$ subunits cause Aicardi-Goutieres syndrome and mimic congenital viral brain infection. Nat Genet 2006;38:910916.

125. Cuadrado E, Vanderver A, Brown KJ, et al. Aicardi-Goutieres syndrome harbours abundant systemic and brain-reactive autoantibodies. Ann Rheum Dis 2014.

126. Hacohen Y, Zuberi S, Vincent A, Crow YJ, Cordeiro N. Neuromyelitis optica in a child with AicardiGoutieres syndrome. Neurology 2015.

127. Ruiz-Gaviria R, Baracaldo I, Castaneda C, Ruiz-Patino A, Acosta-Hernandez A, Rosselli D. Specificity and sensitivity of aquaporin 4 antibody detection tests in patients with neuromyelitis optica: A meta-analysis. Mult Scler Relat Disord 2015;4:345-349.

128. Waters P, Reindl M, Saiz A, et al. Multicentre comparison of a diagnostic assay: aquaporin-4 antibodies in neuromyelitis optica. J Neurol Neurosurg Psychiatry 2016;87:1005-1015.

129. Waters PJ, McKeon A, Leite MI, et al. Serologic diagnosis of NMO: a multicenter comparison of aquaporin-4-IgG assays. Neurology 2012;78:665-671; discussion 669.

130. Crow YJ, Vanderver A, Orcesi S, Kuijpers TW, Rice GI. Therapies in Aicardi-Goutieres syndrome. Clin Exp Immunol 2014;175:1-8. 